\title{
ON A QUESTION OF SÁRKOZY AND SÓS FOR BILINEAR FORMS
}

\author{
JAVIER CILLERUELO AND JUANJO RUÉ
}

\begin{abstract}
We prove that if $2 \leq k_{1} \leq k_{2}$, then there is no infinite sequence $\mathcal{A}$ of positive integers such that the representation function $r(n)=\#\left\{\left(a, a^{\prime}\right): n=k_{1} a+k_{2} a^{\prime}, a, a^{\prime} \in \mathcal{A}\right\}$ is constant for $n$ large enough. This result completes previous work of Dirac and Moser for the special case $k_{1}=1$ and answers a question posed by Sárkozy and Sós.
\end{abstract}

\section{Introduction}

Given an infinite sequence of positive integers $\mathcal{A}$, the representation functions $r(n)$ and $R(n)$ are defined as the number of solutions of the equations

$$
\begin{aligned}
& n=a+a^{\prime}, a, a^{\prime} \in \mathcal{A} \\
& n=a+a^{\prime}, a, a^{\prime} \in \mathcal{A}, a \leq a^{\prime},
\end{aligned}
$$

respectively.

It is obvious that $r(n)$ is odd when $n=2 a, a \in \mathcal{A}$, and even otherwise. So it is not possible for $r(n)$ to be constant for $n$ large enough. This asymmetry disappears in $R(n)$ but Dirac [1] gave a beautiful argument that also proves that $R(n)$ cannot be constant for $n$ large enough.

For $k \geq 2$, Moser [3] considered the representation function

$$
r(n)=\#\left\{\left(a, a^{\prime}\right), n=a+k a^{\prime}, a, a^{\prime} \in \mathcal{A}\right\} .
$$

Surprisingly he constructed a sequence $\mathcal{A}$ such that $r(n)=1$ for all $n \geq 0$.

Sárkozy and Sós [4] asked for which $\left(k_{1}, k_{2}\right)$ the representation function

$$
\left.r_{k_{1}, k_{2}}(n, \mathcal{A})\right)=\#\left\{\left(a, a^{\prime}\right): n=k_{1} a+k_{2} a^{\prime}, a, a^{\prime} \in \mathcal{A}\right\} .
$$

can be constant for $n$ large enough. We answer this question by showing that the only cases with affirmative answer are those considered by Moser.

TheOrem 1.1. Let $k_{1}, k_{2}, 2 \leq k_{1} \leq k_{2}$. Then there is no infinite sequence of positive integers $\mathcal{A}$ such that

$$
r_{k_{1}, k_{2}}(n, \mathcal{A})=\#\left\{\left(a, a^{\prime}\right): n=k_{1} a+k_{2} a^{\prime}, a, a^{\prime} \in \mathcal{A}\right\}
$$

is constant for $n$ large enough.

The question posed in [4] actually concerns general linear forms $k_{1} x_{1}+\cdots+k_{h} x_{h}, h \geq 2$. The same arguments we use for the case $h=2$ can be extended to the general case when the $k_{i}^{\prime} s$ are pairwise coprimes but they are best illustrated in the situation presented in this paper. The more general case with arbitrary coefficients requires a different approach and it is considered in a forthcoming paper.

2000 Mathematics Subject Classification 11B34.

This work was developed during the Doccourse in Additive Combinatorics held in the Centre de Recerca Matemàtica from January to March 2008. Both authors are extremely grateful for its hospitality. 
2. Translation of the problem into generating functions: Dirac's and Moser's arguments

As Dirac and Moser did, we use the language of generating functions: to every set $\mathcal{A}$ of nonnegative integers, we write the formal power series $f_{\mathcal{A}}(z)$ defined as

$$
f_{\mathcal{A}}(z):=f(z)=\sum_{a \in \mathcal{A}} z^{a}
$$

This formal power series is called the generating function associated to $\mathcal{A}$.

For every set $\mathcal{A}$, the corresponding generating function defines an analytic function around $z=0$. This analytic function is a polynomial if $\mathcal{A}$ is finite and has a singularity at $z=1$ if $|\mathcal{A}|$ is infinite. In fact, if $|\mathcal{A}|$ is infinite, then the Taylor expansion around $z=0$ defined by the formal power series has radius of convergence $r=1$.

We proceed to translate the general problem into the language of generating functions. The fundamental equation we use is:

$$
f\left(z^{k_{1}}\right) f\left(z^{k_{2}}\right)=\sum_{a, a^{\prime} \in \mathcal{A}} z^{k_{1} a+k_{2} a^{\prime}}=\sum_{n=0}^{\infty} r_{k_{1}, k_{2}}(n, \mathcal{A}) z^{n} .
$$

\subsection{Dirac's argument}

We observe that for the functions $r(n)$ and $R(n)$ we have the relation $r(n)=2 R(n)-\delta(n)$, where $\delta(n)=1$ if $n=2 a$ for some $a \in \mathcal{A}$ and 0 otherwise. By (2.1) we obtain

$$
f^{2}(z)=\sum_{n=0}^{\infty} r(n) z^{n}=2 \sum_{n=0}^{\infty} R(n) z^{n}-\sum_{a \in \mathcal{A}} z^{2 a}
$$

which can be written in the form

$$
f^{2}(z)+f\left(z^{2}\right)=2 \sum_{n=0}^{\infty} R(n) z^{n}
$$

Dirac proved that $R(n)$ cannot be a constant $c$ for $n \geq n_{0}$ with an easy but clever argument: suppose that $R(n)=c$ for $n \geq n_{0}$. Then

$$
f^{2}(z)+f\left(z^{2}\right)=Q(z)+2 c \frac{z^{n_{0}+1}}{1-z}=\frac{P(z)}{1-z},
$$

where $P(z)$ is a polynomial of finite degree with $P(1) \neq 0$. Then we obtain a contradiction by taking the limit for $z \rightarrow-1$ in both sides of the equation: the left hand side of the equality diverges, but the right hand side has a finite limit.

\subsection{Moser's argument}

Moser $[\mathbf{3}]$ studied the case $k_{1}=1, k_{2} \geq 2$. He wondered if for these cases there exists an infinite sequence of nonnegative integers such that $r_{1, k}(n, \mathcal{A})=1$ for all $n \geq 0$. If this is the case, equation (2.1) implies

$$
f(z) f\left(z^{k}\right)=\sum_{n \geq 0} z^{n}=\frac{1}{1-z} .
$$

If we make the change of variables $z:=z^{k}$ we get

$$
f\left(z^{k}\right) f\left(z^{k^{2}}\right)=\frac{1}{1-z^{k}} .
$$


Dividing the initial equation by this one we obtain

$$
f(z)=\frac{1-z^{k}}{1-z} f\left(z^{k^{2}}\right)=\left(1+z+z^{2}+\cdots+z^{k-1}\right) f\left(z^{k^{2}}\right) .
$$

By iterating we get the relation

$$
f(z)=\prod_{j=0}^{\infty}\left(1+z^{\left(k^{2}\right)^{j}}+z^{2\left(k^{2}\right)^{j}}+\cdots+z^{(k-1)\left(k^{2}\right)^{j}}\right) .
$$

This product defines an analytic function at the origin, which can be written using its series expansion around $z=0$. Moreover, by the unique $k^{2}$-adic representation of an integer, the Taylor's coefficients of $f(z)$ are either 0 or 1 . So this function $f(z)$ defines a set $\mathcal{A}$ which satisfies our assumptions.

More precisely, the set $\mathcal{A}$ is the set of all nonnegative integers such that all its digits in its $k^{2}$-adic expansion are smaller than $k$.

\subsection{The general case}

We want to know if, given $k_{1}, k_{2}, 1 \leq k_{1} \leq k_{2}$, there exists an infinite sequence of non negative integers $\mathcal{A}$ and a value (say $\left.n_{0}\right)$ such that $r_{k_{1}, k_{2}}(n, \mathcal{A})$ is a positive constant $c$ for $n \geq n_{0}$. Since the cases $k_{1}=1$ have been considered by Dirac $\left(k_{2}=1\right.$ with negative answer in both ordered and unordered representations) and Moser $\left(k_{2} \geq 2\right.$ with affirmative answer) we may assume that $2 \leq k_{1} \leq k_{2}$. We may also assume that $\operatorname{gcd}\left(k_{1}, k_{2}\right)=1$, since otherwise we have $r_{k_{1}, k_{2}}(n, \mathcal{A})=0$ for all $n \not \equiv 0\left(\bmod \operatorname{gcd}\left(k_{1}, k_{2}\right)\right)$.

If such a sequence $\mathcal{A}$ exists, then by (2.1) we have

$$
f\left(z^{k_{1}}\right) f\left(z^{k_{2}}\right)=\sum_{n=0}^{n_{0}-1} a_{n} z^{n}+\sum_{n=n_{0}}^{\infty} c z^{n}=Q(z)+\frac{c z^{n_{0}+1}}{1-z}=\frac{P(z)}{1-z}
$$

where $Q(z), P(z)$ are polynomials in $\mathbb{Z}[z]$ with $P(1) \neq 0$. This last relation is equivalent to the condition $c \neq 0$.

For convenience, we take the square of the previous equation. By writing $F(z)=f^{2}(z)$, we want to show that there is no function $F(z)$, analytic in the disc $|z|<1$, such that

$$
F\left(z^{k_{1}}\right) F\left(z^{k_{2}}\right)=\frac{P^{2}(z)}{(1-z)^{2}} .
$$

Theorem 1.1 will be a consequence of a more general theorem:

Theorem 2.1. For any integers $k_{1}, k_{2}, 2 \leq k_{1}<k_{2}$ with $\operatorname{gcd}\left(k_{1}, k_{2}\right)=1$ and any polynomial $P(z) \in \mathbb{Z}[z]$ with $P(1) \neq 0$, there is no function $F(z)$, analytic in the disc $|z|<1$, satisfying

$$
F\left(z^{k_{1}}\right) F\left(z^{k_{2}}\right)=\frac{P^{2}(z)}{(1-z)^{2}} .
$$

In what follows we concentrate in the proof of Theorem 2.1.

\section{Algebraic preliminaries and notation used}

In our work we will use cyclotomic polynomials.

Recall that the cyclotomic polynomial of order $n$ is defined by the relation

$$
\Phi_{n}(z)=\prod_{\xi \in \phi_{n}}(z-\xi) \in \mathbb{Z}[z]
$$


where $\phi_{n}$ denotes the set of primitive roots of order $n$,

$$
\phi_{n}=\left\{\xi \in \mathbb{C}: \xi^{k}=1 \text { if and only if } k \equiv 0 \quad(\bmod n)\right\} .
$$

Many properties of these polynomials are well-known. The most important one for our present purposes is that they are irreducible over $\mathbb{Z}[z]$. As a consequence, it is known that if a polynomial $P(z) \in \mathbb{Z}[z]$ vanishes at a primitive root of order $n$ then there exists a positive integer $s$ such that

$$
P(z)=\Phi_{n}^{s}(z) Q(z)
$$

where $Q(z) \in \mathbb{Z}[z]$ and $Q(\xi) \neq 0$ for all primitive roots $\xi$ of order $n$.

Given $k_{1}, k_{2}, 2 \leq k_{1}<k_{2}$ we write $\xi_{j_{1}, j_{2}}$ to denote a primitive root of order $k_{1}^{j_{1}} k_{2}^{j_{2}}, 0 \leq j_{1}, j_{2}$, and we let $s_{j_{1}, j_{2}}$ denote the nonnegative integer such that

$$
P(z)=\Phi_{k_{1}^{j_{1} k_{2}^{j_{2}}}}^{s_{j_{1}, j_{2}}}(z) P_{j_{1}, j_{2}}(z)
$$

with $P_{j_{1}, j_{2}}(z) \in \mathbb{Z}[z]$ and $P_{j_{1}, j_{2}}(\xi) \neq 0$ for all $\xi \in \phi_{k_{1}^{j_{1}} k_{2}^{j_{2}}}$.

It is not true in general that for an analytic function $F(z)$ with integer coefficients there exists $r_{j_{1}, j_{2}}$ such that

$$
F(z)=\Phi_{k_{1}^{j_{1} k_{2}^{j_{2}}}}^{r_{j_{1}, j_{2}}}(z) F_{j_{1}, j_{2}}(z)
$$

with $\lim _{z \rightarrow \xi} F_{j_{1}, j_{2}}(z) \neq 0, \infty$ for any $\xi \in \phi_{j_{1}, j_{2}}$. However we will prove that there is such a factorization if $F(z)$ satisfies $(2.2)$.

\section{Proof of theorem 2.1}

The proof of Theorem 2.1 is a consequence of the two propositions below:

Proposition 4.1. With the notation used in Section 3, $r_{j, 0}$ and $r_{0, j}$ are well defined for any $j \geq 0$ and they verify the following recurrence relations

$$
\begin{array}{ll}
r_{j+1,0}=2 s_{j+1,0}-r_{j, 0} & \\
r_{0, j+1}=2 s_{0, j+1}-r_{0, j} & \text { (vertical recurrence) }
\end{array}
$$

for any $j \geq 0$, and initial condition $r_{0,0}=-1$.

Proposition 4.2. With the notation used in Section $3, r_{j_{1}, j_{2}}$ are well defined for any $j_{1}, j_{2} \geq 0$ and these numbers verify the recurrence relation

$$
r_{j_{1}-1, j_{2}}+r_{j_{1}, j_{2}-1}=2 s_{j_{1}, j_{2}} \quad \text { (diagonal recurrence) }
$$

for $j_{1} \geq 1, j_{2} \geq 1$.

Before proving the above propositions we show how Theorem 1.1 can be deduced from them.

Since $r_{0,0}=-1$ is an odd number, from the two propositions above we see that all values $r_{j_{1}, j_{2}}$ are odd numbers.

As $P(z)$ is a polynomial, it is clear that $s_{j_{1}, j_{2}}=0$ when $j_{1}+j_{2} \geq j$ for a suitable $j$.

Using proposition 4.1 and for this value of $j$, we obtain the relations:

$$
\begin{aligned}
& r_{j+1,0}=-r_{j, 0} \quad \text { and } \\
& r_{0, j+1}=-r_{0, j} .
\end{aligned}
$$

From proposition 4.2 we get that $r_{j+1,0}=-r_{j, 1}=r_{j-1,2}=\cdots$, so

$$
\begin{aligned}
r_{j+1,0} & =(-1)^{j+1} r_{0, j+1} \quad \text { and } \\
r_{j, 0} & =(-1)^{j} r_{0, j} .
\end{aligned}
$$


The above relations are possible if and only if $r_{j, 0}=r_{j+1,0}=r_{0, j}=r_{0, j+1}=0$, giving a contradiction. This proves Theorem 2.1 and Theorem 1.1.

We next prove the two propositions. In what follows all the limits considered are taken along a radius.

Proof of proposition 4.1. We deal only with the horizontal recurrence. The proof for the vertical recurrence is similar.

We write $r_{j}=r_{j, 0}, s_{j}=s_{j, 0}$ and $\xi_{j}=\xi_{j, 0}$ for simplicity. We shall prove by induction that all the $r_{j}$ 's are well defined.

By writing $F(z)=F_{0}(z) /(z-1)$ in $(2.2)$ we have

$$
F_{0}\left(z^{k_{1}}\right) F_{0}\left(z^{k_{2}}\right)=P^{2}(z)\left(1+z+\cdots z^{k_{1}-1}\right)\left(1+z+\cdots z^{k_{2}-1}\right) .
$$

For $z \rightarrow 1$ the left hand side of the above equation clearly goes to $F_{0}^{2}(1)$ and the right hand side is neither 0 nor $\infty$. Since $\Phi_{1}(z)=z-1$ we obtain that $r_{0}=-1$.

Assume now that $r_{j}$ is well defined. Then

$$
F(z)=\Phi_{k_{1}^{j}}^{r_{j}}(z) F_{j}(z)
$$

where $F_{j}(\xi) \neq 0, \infty$ for all $\xi \in \phi_{k_{1}^{j}}$. Now we write

$$
F(z)=\Phi_{k_{1}^{j+1}}^{2 s_{j+1}-r_{j}}(z) F_{j+1}(z) .
$$

In what follows, we prove that $\lim _{z \rightarrow \xi_{j+1}} F_{j+1}(z) \notin\{0, \infty\}$ for any $\xi \in \phi_{k_{1}^{j+1}}$. This will show that $r_{j+1}$ exists and that $r_{j+1}=2 s_{j+1}-r_{j}$.

We use (4.1) in $F\left(z^{k_{1}}\right)$ and (4.2) in $F\left(z^{k_{2}}\right)$ to write the equation (2.2) in the form

$$
F_{j+1}\left(z^{k_{2}}\right)=\frac{P_{j+1}^{2}(z) \Phi_{k_{1}^{j+1}}^{2 s_{j+1}}(z)}{(1-z)^{2} \Phi_{k_{1}^{j+1}}^{2 s_{j+1}-r_{j}}\left(z^{k_{2}}\right) F_{j}\left(z^{k_{1}}\right) \Phi_{k_{1}^{j}}^{r_{j}}\left(z^{k_{1}}\right)} .
$$

By making the substitution $z=\xi_{j+1} \omega$ we have

$$
\begin{aligned}
F_{j+1}\left(\xi_{j+1}^{k_{2}} \omega^{k_{2}}\right)= & \frac{P_{j+1}^{2}\left(\xi_{j+1} \omega\right)}{\left(1-\xi_{j+1} \omega\right)^{2}} \cdot \frac{1}{F_{j}\left(\xi_{j+1}^{k_{1}} \omega^{k_{1}}\right)} \\
& \cdot \frac{\Phi_{k_{1}^{j+1}}^{2 s_{j+1}}\left(\xi_{j+1} \omega\right)}{\Phi_{k_{1}^{j+1}}^{2 s_{j+1}-r_{j}}\left(\xi_{j+1}^{k_{2}} \omega^{k_{2}}\right) \Phi_{k_{1}^{j}}^{r_{j}}\left(\xi_{j+1}^{k_{1}} \omega^{k_{1}}\right)} .
\end{aligned}
$$

We let $\omega \rightarrow 1$ and we observe that all the primitive roots of order $k_{1}^{j+1}$ can be written in the form $\xi_{j+1}^{k_{2}}$ for a suitable $\xi_{j+1}$ since $\operatorname{gcd}\left(k_{2}, k_{1}\right)=1$.

To conclude, we show that the limit is neither 0 nor $\infty$. In fact, this is the case for each of the three factors on the right hand side of (4.3).

It is clear that $P_{j+1}\left(\xi_{j+1}\right) \neq 0$ by definition.

Since $\xi_{j+1}^{k_{1}} \in \phi_{k_{1}^{j}}$, we use the induction hypothesis to conclude that the limit of the second factor is neither 0 nor $\infty$.

To study the third factor when $\omega \rightarrow 1$ it suffices to analyze the factors in the cyclotomic polynomials which vanish at $\omega=1$. It should be noticed that $\xi_{j+1}^{k_{1}} \in \phi_{k_{1}^{j}}$ and $\xi_{j+1}^{k_{2}} \in \phi_{k_{1}^{j+1}}$. The contribution of these factors is

$$
\begin{gathered}
\frac{\left(\xi_{j+1} \omega-\xi_{j+1}\right)^{2 s_{j+1}}}{\left(\xi_{j+1}^{k_{2}} \omega^{k_{2}}-\xi_{j+1}^{k_{2}}\right)^{2 s_{j+1}-r_{j}}\left(\xi_{j+1}^{k_{1}} w^{k_{1}}-\xi_{j+1}^{k_{1}}\right)^{r_{j}}} \\
=\frac{\xi_{j+1}^{2 s_{j+1}}}{\xi_{j+1}^{k_{2}\left(2 s_{j+1}-r_{j}\right)} \xi_{j+1}^{k_{1} r_{j}}} \cdot \frac{(\omega-1)^{2 s_{j+1}}}{\left(\omega^{k_{2}}-1\right)^{2 s_{j+1}-r_{j}}\left(\omega^{k_{1}}-1\right)^{r_{j}}}
\end{gathered}
$$


which tends to

$$
\frac{\xi_{j+1}^{2 s_{j+1}\left(1-k_{2}\right)+r_{j}\left(k_{2}-k_{1}\right)}}{k_{2}^{2 s_{j+1}-r_{j}} k_{1}^{r_{j}}}
$$

as $\omega \rightarrow 1$. The relevant fact about this limit is that it is neither zero nor infinity.

The next proof is quite similar to the previous one:

Proof of proposition 4.2. We will prove, for each diagonal $j_{1}+j_{2}=j$, that all $r_{j_{1}, j_{2}}, 0 \leq$ $j_{2} \leq j$ are well defined. For each $j$ we will do it by induction on $j_{2}$.

This is true for $r_{j, 0}$ by Proposition 4.1. Suppose that $r_{j_{1}, j_{2}}$ is well defined. Thus

$$
F(z)=\Phi_{j_{1}, j_{2}}^{r_{j_{1}, j_{2}}}(z) F_{j_{1}, j_{2}}(z)
$$

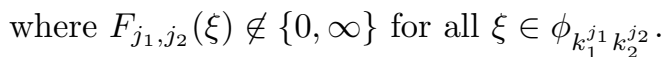

We prove that $r_{j_{1}-1, j_{2}+1}$ is also well defined and also that $r_{j_{1}-1, j_{2}+1}=s_{j_{1}, j_{2}+1}-r_{j_{1}, j_{2}}$.

In order to do this we write

$$
F(z)=\Phi_{j_{1}-1, j_{2}+1}^{2 s_{j_{1}, j_{2}+1}-r_{j_{1}, j_{2}}}(z) F_{j_{1}-1, j_{2}+1}(z) .
$$

What we have to prove is that $\lim _{z \rightarrow \xi} F_{j_{1}-1, j_{2}+1}(z) \notin\{0, \infty\}$ for any $\xi \in \phi_{j_{1}-1, j_{2}+1}$.

We use (4.4) in $F\left(z^{k_{2}}\right)$ and (4) in $F\left(z^{k_{1}}\right)$ to write the equation (2.2) in the form

$$
F_{j_{1}-1, j_{2}+1}\left(z^{k_{1}}\right)=\frac{P_{j_{1}, j_{2}+1}^{2}(z) \Phi_{j_{1}, j_{2}+1}^{2 s_{j_{1}, j_{2}+1}}(z)}{(1-z)^{2} \Phi_{j_{1}-1, j_{2}+1}^{2 s_{j_{1}, j_{2}+1}-r_{j_{1}, j_{2}}}\left(z^{k_{1}}\right) \Phi_{j_{1}, j_{2}}^{r_{j_{1}, j_{2}}}\left(z^{k_{2}}\right) F_{j_{1}, j_{2}}\left(z^{k_{2}}\right)} .
$$

Now we make the substitution $z=\xi_{j_{1}, j_{2}+1} \omega$ for some arbitrary $\xi_{j_{1}, j_{2}+1} \in \phi_{k_{1}^{j_{1}} k_{2}^{j_{2}+1}}$. We obtain the expression

$$
\begin{array}{r}
F_{j_{1}-1, j_{2}+1}\left(\xi_{j_{1}, j_{2}+1}^{k_{1}} \omega^{k_{1}}\right)=\frac{P_{j_{1}, j_{2}+1}^{2}\left(\xi_{j_{1}, j_{2}+1} \omega\right)}{\left(1-\xi_{j_{1}, j_{2}+1} \omega\right)^{2}} \cdot \frac{1}{F_{j_{1}, j_{2}}\left(\xi_{j_{1}, j_{2}+1}^{k_{2}} \omega^{k_{2}}\right)} \\
\cdot \frac{\Phi_{j_{1}, j_{2}+1}^{2 s_{j_{1}, j_{2}+1}}\left(\xi_{j_{1}, j_{2}+1} \omega\right)}{\Phi_{j_{1}-1, j_{2}+1}^{2 s_{j_{1}, j_{2}+1}-r_{j_{1}, j_{2}}}\left(\xi_{j_{1}, j_{2}+1}^{k_{1}} \omega^{k_{1}}\right) \Phi_{j_{1}, j_{2}}^{r_{j_{1}, j_{2}}}\left(\xi_{j_{1}, j_{2}+1}^{k_{2}} \omega^{k_{2}}\right)}
\end{array}
$$

Now we let $\omega \rightarrow 1$. We observe that all the primitive roots of order $k_{1}^{j_{1}-1} k_{2}^{j_{2}-1}$ can be written in the form $\xi_{j_{1}, j_{2}+1}^{k_{1}}$ for a suitable $\xi_{j_{1}, j_{2}+1} \in \phi_{k_{1}^{j_{1}} k_{2}^{j_{2}+1}}$. As in the previous proposition, we show that the limit is neither 0 nor $\infty$, showing that this is the case for every factor in the previous equation.

For the first factor, it is clear that $P_{j_{1}, j_{2}+1}\left(\xi_{j_{1}, j_{2}+1}\right) \notin\{0, \infty\}$ by definition.

Since $\xi_{j_{1}, j_{2}+1}^{k_{1}} \in \phi_{k_{1}^{j_{1}-1} k_{2}^{j_{2}+1}}$, we use induction hypothesis to conclude that the limit in the second factor does not belong to $\{0, \infty\}$.

Finally, to study the third factor when $\omega \rightarrow 1$ we look at the cyclotomic polynomials which vanish at $\omega=1$. It should be noticed that $\xi_{j_{1}, j_{2}+1}^{k_{1}} \in \phi_{k_{1}^{j_{1}-1} k_{2}^{j_{2}+1}}$ and $\xi_{j_{1}, j_{2}+1}^{k_{2}} \in \phi_{k_{1}^{j_{1}} k_{2}^{j_{2}}}$. The contribution of these factors is

$$
\frac{\left(\xi_{j_{1}, j_{2}+1} \omega-\xi_{j_{1}, j_{2}+1}\right)^{2 s_{j_{1}, j_{2}+1}}}{\left(\xi_{j_{1}, j_{2}+1}^{k_{1}} \omega^{k_{1}}-\xi_{j_{1}, j_{2}+1}^{k_{1}}\right)^{2 s_{j_{1}, j_{2}+1}-r_{j_{1}, j_{2}}}\left(\xi_{j_{1}, j_{2}+1}^{k_{2}} \omega^{k_{2}}-\xi_{j_{1}, j_{2}+1}^{k_{2}}\right)^{r_{j_{1}, j_{2}}}} .
$$

which tends to a number which is neither zero nor infinity. This concludes the proof.

\section{References}

1. G. A. Dirac, 'Note on a Problem in Additive Number Theory', J. London Math. Soc. 26 (1951) pp. 312-313. 
2. P. Erdős, P. TurÁn, 'On a problem of Sidon in Additive Number Theory, and on some related problems', J. London Math. Soc. 16 (1941) pp. 212-215.

3. L. Moser, 'An Application of Generating Series', Mathematics Magazine (1) 35 (1962) 37-38.

4. A. SÁrkozy, V. T. Sós, 'On additive representation functions', The mathematics of Paul Erdös I (eds P. Erdős, R. L. Graham and J. Nesetril), Algorithms Combin. 13 (Springer, Berlin, 1997), pp 129-150

Javier Cilleruelo

Departamento de Matemáticas Universidad Autónoma de Madrid 28049 Madrid, Spain

franciscojavier.cilleruelo@uam.es
Juanjo Rué

Departament de Matemàtica Aplicada II

Universitat Politècnica de Catalunya

Jordi Girona 1-3

08034 Barcelona, Spain

juan.jose.rue@upc.edu 